\title{
Time-based Signal Processing and Shape in Alternative Rock Recordings
}

Samantha Bennett

The Australian National University

samantha.bennett@anu.edu.au

\begin{abstract}
This article considers the impact of time-based signal processors on the shape of alternative rock recordings. The concept of musical shape is considered here as both textural and gestural. In this instance, texture pertains to the interactions between the component parts of a multitrack recording and gesture relates to applications of processing by the recordist. Drawing on examples by the Jesus and Mary Chain, Siouxsie and the Banshees, Mazzy Star and My Bloody Valentine, the impact of time-based signal processors on the shape of the recordings is elucidated.
\end{abstract}

KEYWORDS: technology, production, recording, reverb, alternative, rock

\section{Research context}

Scholars, including many of those involved in the Art of Record Production forum, ${ }^{1}$ have established the recording studio/workplace, sound recording and production technology, recordists, as well as techniques/process as being the contextual cornerstones of record production study. However, the foregrounding of these factors in music analysis is less common, and to that end my method has focused less on social aspects and reception - that is, how audiences, critics and cultural commentators respond to recordings - and more on the impact of these oft-overlooked factors on the music itself.

In popular musicology and music analysis, work focusing on technological and/or processual matters is still in its infancy, due in part to what Stephen Cottrell (2010) has noted as the relatively recent development of phonomusicological 
analysis as worthy of scholarly enquiry. As such, methods of analysing recordings in terms of their sonically discernible technological and processual interventions, as opposed to the musical make-up of the instrument performances, remain notably absent from popular musicology. There is, however, an increasingly rich source base of work on the wider, contextual issues surrounding audio recording and technology. Richard James Burgess' Art of Music Production (2005) is one example, focusing largely on the role of the recordist and the development of necessary skill sets required to work in the recording industry. Mark Cunningham's Good Vibrations (1998) and Greg Milner's Perfecting Sound Forever (2009) have covered historical issues pertaining to the development of sound recording technologies and processes. Mark Katz' Capturing Sound (2004) notes significant developments of music technologies via a range of examples and eras. However, more specifically, the relevance of technology and electroacoustical properties to musical performances is noted only to an extent in popular musicology. Philip Tagg suggests "acoustical aspects" and "electromusical \& mechanical aspects" (Tagg 2000: 82) as two important factors in his "checklist of musical expression", which feature seven musical elements in total. Tagg's hermeneutic-semiological method ensures that "no important parameter of musical expression is overlooked in analysis" (Tagg 2000: 83). This is certainly a good start and clearly recognises the integral role and subsequent impact of technological intervention on music recordings. More recent - and most relevant to this article - is the work carried out by Allan Moore $(1993,2012)$ and by Ruth Dockwray and Allan Moore (2010). Moore argues that a "virtual performance space" is constructed in a listener's mind when listening to recordings. Moore describes this space as the "sound box", featuring four dimensions: volume, frequency, stereo image and time (1993: 181). Dockwray and Moore's work, whilst essential to the development of studies in this field, focuses on the position of listener. This is indeed an essential point of focus, but the contextual details of workplace, recordist, and technologies are not illuminated. In his in-depth study on popular music analysis and meaning, Song Means (2012), Moore furthers discussion on recording and production using Edward Hall's proxemics as a contextual framework, as he describes:

Proxemics describes and analyses the distances (social, public, private, intimate) between individuals-in-interaction. These are a factor, on recordings, not only of the audible distance of a persona from the listener's position (i.e. loudness and degree of reverberation) but of the degree of congruence between a persona and personic environment. (Moore 2012: 186)

This is an extremely useful methodology for analysing distances created in popular music mixes by mix position, dynamics, and processing. However, what I am interested in here is less the relationship/proximity between persona and listener and more the effect of processing on what is eventually heard.

In 2012, Allan Moore and I presented a paper entitled "Tech-Processual Shaping of Recorded Popular Song" at Kings College, London, presenting findings from some analytical work on Manchester indie band Doves and US electro-pop act MGMT (Bennett and Moore 2012). In this paper, we conducted our analyses 
using a broad methodology loosely based on Hall's aforementioned proxemics (1966) and Mark Johnson's embodied schemata (1987); specifically, the containment schema denoting relationships between inside and outside and spatial motion. These are useful frameworks for conceptualizing the shape of recorded song as it traverses over a period of time. Our analyses took in multiple matters of sonically discernible technological influence on what is eventually heard, including: instrument microphone position; position of instruments in the stereo field; depth of field (that being the position of instruments in relation to the foreground, middle, or rear of the overall mix); dynamic range; manipulation of frequency; and manipulation of time. Processual matters, including the gestures made by the recordist, were also acknowledged and discussed. One of the key findings from our research was that applications of time-based effects processors not only impact recordings in terms of spatial characteristics, but more significantly, in terms of texture and a song's overall shape.

Time-based signal processors can be applied in order to:

- prolong and/or extend a sound in terms of its duration;

- control its density by means of manipulating diffusion;

- contain or release a sound;

- repeat it any given number of times;

- position it in a space alternative to that in which it was originally recorded.

As such, applications of time-based signal processors can impact dramatically on what is eventually heard and, therefore, deserve to be singled out for a more focused study such as this one. Scholars including Izhaki (2008) and Hodgson (2010) recognize time-based signal processors as they are used in the context of overall mixes, but do not single out specific examples for detailed analysis. Some of the most enlightening and useful work on reverb and delay is in the context of Caribbean music, specifically reggae and dub. The centrality of time-based signal processing to such genres, both historically and technically, has been explored in depth by Howard (2016) and Alleyene (2008). As outlined in Bennett (2015), the concept of musical shape is conceptualized here as both textural and gestural. In these instances, texture pertains to the interactions between the component parts of a given multitrack recording, to include recognition of the aforementioned list of applications, and gesture relates to applications of time-based processing by the recordist.

\section{Time-based signal processors}

So why focus on time-based processors? Such systems impact textural aspects of a recording perhaps more so than any other processor. As Dockwray and Moore (2010) realized, the four main dimensions of a recording can be described as the stereo field (which can be perceived as left to right), dynamics (loud to quiet), frequency spread (high to low) and time (the temporal aspects of the instruments and how they change over time). In this study, I am not so much interested in the frequency/spatial qualities of these recordings (although there are definitely relationships between time-based processing and these other recording dimensions), but in the various ways time-based signal processing creates, alters, 
and manipulates components of a recording, thus affecting its overall texture and, in turn, its shape.

By time-based processing, I am interested in what Peter Doyle calls "the fabrication of space". Referring to his own experience as a guitarist, Doyle has stated: "the merest touch of echo and reverb could greatly alter the emotive impact of sounds produced and the effective change often seemed to be all out of proportion to the purely sonic changes wrought by the effect" (Doyle 2005: 4). Doyle's book focuses on recorded music pre-1960, and features much insightful work on the application of reverb in 1950s rock and roll. Reverberation, as Doyle notes, was used in pre-1960s recordings to fabricate space, to emulate the acoustics of a room, and/or to evoke a particular place or landscape. In this article, I am concerned with contemporary music and the wider use of time-based processors in the creation and alteration of musical shape. When Doyle touches on the "emotive impact" of echo and reverb, he makes an important point that has also been noted by Nicola Dibben, who states that, "emotional authenticity manifests in sound recordings", "contemporary popular music recording techniques such as reverb, delays, filters and overdubbing help 'stage' voices" (2009: 319).

Whilst Dibben refers to the effect of time-based processors on situating the voice, a recording may feature multiple time-based processors on a single instrument, an instrument group or, indeed, across an entire mix. As Machin points out: "Owing to the association of echo and reverb with space, it can also be used to suggest isolation and loneliness. A singer can be portrayed as alone and isolated in a pop video and the reverb can realize this effect through sound" (Machin 2010: 125-6). This observation suggests that not only can reverb "stage" a voice, but it can apply emotion to that staged voice, alter mood, and lend the voice an all-important persona, which will be discussed in further detail through the case studies.

So what effects are we focusing on here? Time-based processors are so-called because they fabricate, simulate, or otherwise manipulate temporal parameters of sound. In this article I refer to the application of specific processors including echo, ${ }^{2}$ reverberation, ${ }^{3}$ delay, ${ }^{4}$ and chorus. ${ }^{5}$ The ratio between a reverberant signal in relation to its original, unprocessed signal is often referred to in audio engineering as the balance between "wet" (reverberant) and "dry" (original) signal (Bartlett and Bartlett 2012). A relatively wet signal - particularly on instruments including vocals and guitars - has become a widely accepted, common trope of commercial rock and pop record production. The easiest method of creating space in a recording is to situate the instrumentalist in an environmental space during the recording process, thus capturing both the instrument and the acoustic characteristics of the environment simultaneously; a small room will be less reflective than a large hall or cathedral, for instance. However, pre-1960s, the fabrication of space was a common technique in sound recording. If an instrument is recorded in a large hall, for example, there is no way of separating the original instrument out from the hall ambience at the mix stage. The application of time-based processors gave recordists a means of controlling how much ambience to add to a recording. Albin Zak describes ambience thus: "Multiple echoes produced by sound reflecting randomly off surfaces in an 
enclosed space accumulate to form an aural image known as ambience or reverb" (Zak 2001: 76). Serge Lacasse goes further to describe reverb as being both acoustical and mechanical, stating that reverb is "a term used in popular music to designate either an effect of sound prolongation or persistence resulting from successive but imperceptible echoes, or the electromechanical device producing such an effect" (2003: 228).

In the 1960s, this was achieved through the use of echo chambers: small rooms - often stairwells or bathrooms - containing a speaker and a microphone. An instrument or mix would be sent out to the speaker, with the microphone capturing a combination of the speaker output and the ambience/reverberation of that particular space. The microphone signal was then fed back to the control room, allowing the recordist to mix in the echo signal with the original. Albin Zak noted the use of dedicated echo chambers, ADT (Artificial Double Tracking), and early digital effects processors in recordings of the 1960s and 1970s (2001). He defined ADT as "an electronic process that simulates the doubling of a vocal or instrumental part" (2001: 221). This technique, pioneered by recordists at London's Abbey Road studios, involved the use of a tape machine connected to a second speed-controlled tape machine in order to double the voice or instrument. Indeed, the presence of time-based signal processing in the repertoire of the Beatles is well documented (Everett 2000; Kehew and Ryan 2006), and the development of plate, spring, and dedicated tape machine devices - not only to fabricate space, but to manipulate, automate and transform it - is considered a key turning point in the development of recorded sound, as Zak suggests:

\begin{abstract}
For sound that is produced at a particular location can effectively be "placed" somewhere else through various techniques of ambience generation, such as an "echo chamber", a vibrating steel plate, or computer software. The last has given recordists for the last twenty years an unprecedented array of possible ambient configurations, that is, any sound can be placed anywhere. (Zak 2001: 77)
\end{abstract}

The application of fabricated echo to sound recordings was pioneered by Sam Phillips of Sun Studios. Phillips manipulated the distances between the record and playback heads on analogue tape machines to create the effect, which can be heard on the records of Elvis Presley, Johnny Cash and others on the Sun label. Dedicated machines, such as those made by Echoplex, consolidated this effect in a small system, eliminating the need for multiple stand-alone tape recorders. By the 1960s, large plate reverberators such as the EMT 140 (released in 1957), were used to create hollow, "glassy" sounding reverbs entirely manipulated by sending audio signals through a $1 / 4$-ton plate of steel. Low cost spring reverberators worked in much the same way, by sending an audio signal through a large metal spring featuring a transducer at one end of the coil and a pickup at the other. Whilst plate and spring reverbs of the 1960s and 1970s provided workplaces with a viable means of fabricating space in post-production, the impact on recorded popular music would be more fully realized in the 1980s with the proliferation of digital reverberation effects. Multiple technological developments in the late 1970 s resulted in emergent digital effects processors that would transform the potential of sound manipulation. This is not an overstatement; following the 
advent of the Eventide H910 Harmonizer in the mid 1970s, for example, recordist Tony Visconti elucidated its transformative potential on David Bowie's Low (1977), its pitch-shifting capability audible on tracks such as "Subterraneans". Visconti himself described the sound as "radical" (Uncut 1999) and also acknowledged the reproduction of similar sounds integrated into cheaper devices as a direct result of the Eventide's popularity. The H910 Harmonizer was also used to significant effect on the Sex Pistols' album Never Mind the Bollocks... (1977), specifically on the vocals to "Sub Mission" (Bennett 2015).

The use of these four processors - the Lexicon 224, EMT 250, Eventide Harmonizer and AMS Digital Reverberator and delay units - by elite recordists and workplaces in the commercial recording industry proliferated throughout the 1980s. These processors were expensive and out of reach to most musicians and semi-professional recordists, and became part of an elite pantheon of aspirational and often unattainable sonic tropes. Since the 1970s, digital reverberators have emulated spaces; rooms, halls and cathedrals are common settings, often featuring adjustable temporal parameters such as repeats, decay, and diffusion. These space emulations on digital reverberators are common settings and feature in many types of recorded popular music since. Whilst the Lexicon 224, AMS and Eventide units became sonic hallmarks of 1980s commercial popular music, little is documented about their impact on the music of the era.

The creative application of time-based signal processing is certainly embedded in the music of the Beatles, as well as artists such as Jimi Hendrix, Miles Davis, Pink Floyd, and Funkadelic. The effects heard in the work of such artists of the 1960 s and 1970 s paved the way for innovative applications of time-based processing in the 1980s and 1990s. During these decades, such processors were used in increasingly creative ways, impacting on musical elements including tempo, texture, and harmony like never before.

So why focus on alternative rock recordings? The post punk era - in both the United Kingdom and the United States - leading up to and at the turn of the 1990 s is an ideal, yet understudied, era in popular music analysis. Set against the backdrop of the conservative, performance-led techniques of 1970s commercial rock production, as well as the large-format console-driven popular music construction approaches of the 1980s (Bennett 2009), post-punk and music labelled "alternative" can be viewed as a reaction to/resistance against the commercial popular music mainstream. As such, the music of this era and genre is packed full of interesting - even unorthodox - examples of production, disconnected from those common to the mainstream. I have studied the use of time-based processing on the music of The Cure, Joy Division, Siouxsie and the Banshees, Mazzy Star and The Jesus and Mary Chain, among others. These artists often foreground the use of time-based signal processors in their music, and as such the effects are not only easily sonically discernible but intrinsic to their very musical aesthetic. Additionally, many of these recordings were made in independent and/or semi-professional recording workplaces. By the late 1980s, the tools of the 1960s and 1970s elite workplaces and recordist guild had become available to semi-professional studios, recordists, and technology-aware musicians, resulting in an altogether different kind of application. 
To that end, focusing on four examples of predominantly independent recordings featuring prominent time-based signal processing is useful for multiple reasons:

- the potential for time-based signal processors to create, simulate, transform, and mask musical elements, and therefore to impact the recording's overall shape, is elucidated;

- the nature of time-based signal processors as intrinsic to musical identities is discussed;

- time-based signal processing as an artist's sonic signature - equal to, or in some instances greater than, instrumental prominence - is also recognized;

- the manifestation of elite 1960s and 1970s tools in 1980s and 1990s alternative music is acknowledged.

\section{The Jesus \& Mary Chain, "Just Like Honey", Psychocandy, 1985}

And so on with the case studies. Firstly, let's focus on "Just Like Honey" by UK alternative act The Jesus and Mary Chain. Taken from the album Psychocandy, this track was recorded by John Loder at Southern Studios in Wood Green, London. The use of reverberation in independent guitar-based musics has been acknowledged, notably by Matthew Bannister:

Reverberation also has the effect of covering up mistakes, an important consideration for relatively amateur musicians. It was used extensively by UK indie acts (The Smiths, The Jesus and Mary Chain, My Bloody Valentine, Spacemen 3) and US groups REM and Hüsker Dü. (Bannister 2006b: 74)

Bannister goes on to acknowledge the clear allusion to Phil Spector's "wall of sound" sonic signature in such music, suggesting that "jangle and drone plus reverberation create a contemporary equivalent of Spector's 'wall of sound' - a massive, ringing, cavernous noise" (2006b: 74).

On "Just Like Honey", we hear multiple similarities to Spector's wall of sound technique and, with that in mind, the first two minutes of the track are the point of focus here. Firstly, the kick drum pattern in the opening two bars is almost identical to one of Spector's most lauded recordings, the Ronettes' "Be My Baby" (1963). As discussed in Bennett (2016), Southern Studios was home to two key time-based signal processors: an EMT 140 plate and an AMS RSX-16 digital reverb. On this track, the opening drums are treated with a plate setting featuring a lengthy decay time of more than 1.5 seconds. It is possible to ascertain this as the reverberant tails from the kick and snare/tambourine pattern do not fully decay prior to the following hit. This suggests that the damper on the EMT Plate was set towards its maximum distance. Undoubtedly, this effect situates the musicians in an enormous, cavernous space as Bannister described. However, the effect is so prominent that the instruments and melodic lines are heard beneath it, as opposed to above or in front of it. This has significant implications for the track's texture: as the introduction progresses and the drums are joined by a relatively dry bass guitar (00:05) and heavily reverberant guitar (00:09), the track's empty and hollow opening shape is steadily filled. As the lead vocal enters (00:17), also featuring a lengthy plate reverb, the track's instrumental components 
are submerged under layers of lengthy, rich reverbs, thus foregrounding the (fabricated) location and space the musicians are situated in, as opposed to the musical progression. This combination of kick drum, snare/tambourine, bass, guitar and vocal holds the track's shape until just prior to the bridge, where a second reverberant guitar overdub enters at 00:40, further filling out the track's mid range. Here, the addition of yet more reverb adds more depth and the stationary, mono position of the instruments and reverb only adds to the track's textural density: no attempt has been made to situate or separate instruments away from each other in order to clarify or highlight them. This is further exacerbated at 00:56, when the original guitar moves from a quarter note to an eighth note arpeggio, resulting in a perceived overall increase in tempo, as well as generating further layers of reverb. Yet more textural density is created at 01:27, where a second snare hit is added to the rhythm progression and another reverberant guitar is overdubbed and foregrounded in the mix.

It is not until 01:51 that the impact of this gradual building of dense, reverberant layers is fully realized in terms of the track's overall shape. At this point, the overdubbed guitars and bass drop out and the drums return to the original pattern against the lead vocal. Here, the track's original, cavernous shell is dramatically exposed after multiple reverb layers decay.

In this track, the deliberate submergence of instrumentation below an effects processor cannot be attributed to simply covering up mistakes. Even though timing issues are audible throughout, the swamping of instruments in lengthy reverb is a definite, sonic statement. For the Jesus and Mary Chain, reverb is a bold signature motif as opposed to an attempt to recreate room ambience. This will be addressed further in the discussion at the end of the paper.

\section{Siouxsie and the Banshees, "The Killing Jar", Peepshow, 1988}

Taken from the 1988 Siouxsie and the Banshees album Peepshow, "The Killing Jar" is a good example of multiple, simultaneous time-based effects processing across a range of instrumentation. In this track, the processing enhances and exemplifies lyrical meanings. The lyrical concept is based around the scientific insect suffocation device as an analogy for a trapped relationship, broadly based on John Fowles' 1963 novel The Collector. Like the entirety of Peepshow, "The Killing Jar" features a range of instrumentation unusual in popular recorded song of the era, including a foregrounded cello, a güiro, chimes, and triangle, as well as standard instruments including drums, bass and guitar. The lead vocals, cello, and triangle have been treated with a lengthy room reverb throughout, resulting in a heavily layered and dense texture. Additionally, the reverberant tails of the vocals are left particularly prominent and sibilant, which is especially audible on words such as "cuts" (00:30). Changes in texture are of course attributable to both the performance elements and processing, however, the application of a prominent chorus effect on both the bass and guitar results in the construction of defined, alternate spaces within the same track. As Kahlin and Ternström pointed out: 
Indeed, this classical device has almost assumed an identity of its own, to the point where the term "chorus effect" is likely to be understood as the sound of three similar signals added together, each with a slowly oscillating time delay that is 120 degrees out of phase with the others. (Kahlin and Ternström 1999: 75)

Kahlin and Ternström are right to highlight the strong identity of this effect: a chorus effect can significantly impact on the temporal parameters of a given sound, multiplicating, lengthening and broadening the instrument to which it is applied. In "The Killing Jar", this has been used to creative effect not only to alter the shape of the overall track but also to create alternate spaces within it.

A notable section lies in the difference between the first and last eight bars of verse one (00:15-00:56): at 00:42, the cello rhythm changes from eighth notes to staccato sixteenth notes against the güiro and an increasingly busy, percussionheavy drum backdrop. This results in a perceived increase in tempo and subsequently creates a sense of anticipation and urgency as the track progresses towards the bridge. In this track, the instruments are positioned around the stereo field, resulting in a wide, almost panoramic soundscape. A heavily chorused bass guitar fills out the track's overall shape in terms of both depth and width, as it creates a broad, swirling whirlpool-like motion. This circular motion is enhanced further by the guitar, which features both a chorus and lengthy reverb. The staccato chord chucks played throughout the introduction on each third beat do not fully decay before the next chord is played. However, by 00:16 the effects are removed from the guitar, presumably to make room in this already quite swampy mix for the vocal.

The bass guitar is perhaps the most significant instrument in this track as it is pivotal in the alteration of overall shape by the second verse (01:37). Here the guitar, cello, and percussion drop out and the drums are positioned to the rear of the mix, leaving a prominent bass guitar and vocal in the foreground. The bass guitar's heavily chorused "swirling" motion is now exposed, and whilst the instrument features chorus throughout, it is foregrounded here due to the absence of the cello and guitar. Rather than add textural depth (as it does earlier and later in the track), when isolated it is tight, up-front and contained. Chorus has also been applied to the vocal's reverberant tails creating long, wispy decays which are particularly effective on sibilant words such as "soft" (01:38) and "shadow" (01:39). This has a dramatic overall effect on the track's shape as the exposed bass guitar suddenly appears trapped in a much smaller, contained space, thus perfectly alluding to the track's central lyrical theme, that of the "killing jar". As the instruments return to the mix, a reverberant chime is heard on both "glass hand" (01:51) and "water" (01:54). Here, the first chime's reverberant tail has not fully decayed when the second one sounds, creating a rippling, shimmering effect. Again, the lyrical narrative takes place near a body of water; the positioning of these reverberant chimes enacts and amplifies the meaning of the lyrics (Moore 2012: 196). Further consideration is given to the various aforementioned time-based signal processing in the discussion at the end of the paper. 


\section{Mazzy Star, "Blue Flower", She Hangs Brightly, 1990}

Little to nothing is known about the context in which Mazzy Star recorded their debut album, She Hangs Brightly. Most of their two follow up albums - So Tonight That I Might See (1993) and Among My Swan (1996) - were recorded at Live Oak studios, a commercial facility in Los Angeles. What is known is that guitarist David Roback, formerly of 1980s groups Rain Parade and Opal, is cited as producer and has spoken, albeit sporadically and vaguely, about recording Mazzy Star; a duo of Roback on guitar and Hope Sandoval on vocals. Due to Roback's prior affiliation with Rain Parade, it is very likely that She Hangs Brightly was recorded at Radio Tokyo, a small recording facility in Venice, California. By the late 1980s, the studio featured a 24-track tape machine as well as new and vintage outboard effects processors. The key reverb machine at Radio Tokyo was a Lexicon PCM-70; a digital time-based signal processor featuring more than forty parameters (Crane 2009: 37).

Whilst "Blue Flower" is a cover of a song originally recorded by avant-rock band Slapp Happy, this version bears little resemblance to the original. Additionally, this is not the only intertextual aspect to the track; the overall mix, genre characteristics, and performance, as well as the prominent foregrounding of time-based signal processors, strongly allude to the Velvet Underground, specifically The Velvet Underground and Nico (1967).

The introduction is particularly notable for its (initially) isolated guitar; aside from a small amount of reverb at source (either via a pedal or amplifier), the guitar is kept relatively dry. At 00:09, a snare roll featuring a lengthy hall reverb enters. At this point, we hear the drums situated in an entirely separate space to the initial guitar. Since it is unlikely that these two instruments were recorded in separate spaces, we can deduce that the reverb applied to the drums is fabricated and, due to its transparent and slightly detached position, it is most likely a hall reverb setting from the Lexicon. By 00:10, a bass guitar, second guitar, and full drum kit enter along with a tambourine. Here, the combination of slow track tempo with thick textured, reverberant drums and strummed, distorted guitars has created a dense, heavy musical backdrop. To an extent, the tambourine balances out the track in terms of its frequency content. However, the lengthy hall reverb applied to it (as well as the nonchalant performance technique) extend it into a constant, almost looped presence. As such, the tambourine is assimilated into the track's thick, dense texture as opposed to adding embellishment. At times, particularly during the introduction, percussive instruments including the snare drum, hand claps, and tambourine appear indistinguishable. This is a good example of a track where the shape simply increases in density with every additional, reverberant layer.

One notable aspect to "Blue Flower", as indeed to Mazzy Star's entire repertoire, is Hope Sandoval's vocal performance and, particularly, the use of time-based signal processors as a signature motif. The reverb is so prominent and so intrinsic to Sandoval's overall musical and performance aesthetic that it is difficult to conceive of her vocal without it. Again, we can deduce from the sound of the reverb that it is fabricated; whilst the space constructed for the vocal appears to be that of a subway or other enclosed, reflective space, the processing is tightly controlled. After a lengthy introduction, the vocal enters at 00:38. Here, 
more than one time-based signal processor is heard. Firstly, a short echo or delay has been applied - a common technique designed to add depth to a vocal performance. This is particularly audible on prominent words such as "forgotten" (00:47) and "walking" (00:58). Secondly, a large hall reverb - similar to that heard on the snare drum - has also been applied. However, unlike the snare drum, which features lengthy reverberant tails, the vocal reverb tails are much shorter. This has created a dramatic sounding space for Sandoval's vocal: the voice is staged in a large, reflective space, but the reverb decays quickly, thus not crowding it. This creates a sense of detachment, as if the vocals are being performed in a bubble. This use of time-based signal processing - coupled with the position of the vocals in the mix, enveloped by two distorted guitars - results in a heavy and mushy overall shape where instrument performances have blended and merged under swathes of reverberant layers.

\section{My Bloody Valentine, "I Only Said", Loveless, 1991}

As one of the most sonically distinctive artists in independent rock history, it is unsurprising that UK shoegaze act My Bloody Valentine are also the most studied of the case studies here (McGonigal 2007; Blake 2012; McDonald 2000; Sangild 2011). The track "I Only Said" is taken from the 1991 album Loveless, famously recorded over a two-year period at various studios by singer/guitarist Kevin Shields and recordist Alan Moulder, as well as other assistant engineers. Whilst the presence and politics of noise in the group's work has been acknowledged by scholars including McDonald (2000), Sangild (2011) and Blake (2012), their use of time-based processing has not. Sangild (2011: web source) states in reference to Loveless: "The guitar chords are gliding, swimming in a muddy sea of distortion. The guitarists' strokes are cut off in the mixing process, so that every sound seems to be growing out of nowhere, with no distinct edges". This impenetrable, textured layering of multiple noise-laden guitar sounds is so dense as to render the instruments indistinguishable from each other. The overall production, as Blake (2012: web source) quite rightly points out, "shrouds its pitch characteristics under a timbral wash". This "cutting off" of guitars that Sangild refers to is particularly prominent in "I Only Said", and has been achieved with a particularly unusual method of reverse, gated reverb. As Kevin Shields explains,

And the one massive, big effect that we used a lot was a thing called reverse reverb, as well as reverse gated reverb. By having that effect on full and by taking all the original guitar tone off, it allowed me to create this ultra-melted guitar sound. (Shields, quoted in McGonigal 2007: 50)

The reverse reverb Shields describes was achieved via a parameter on the Yamaha SPX-90, a budget recording studio and live audio multi-effects processor. The time-based signal processors on the SPX-90 - particularly the hall and room settings - are ubiquitous in both recorded and live music. However, Shields' use of the processor was somewhat unorthodox, as the reverse reverb on the Yamaha SPX-90, "inverts a normal reverb envelope without making the notes backwards. There are certain settings I use that ... create a liquid sound. I don't use any of the original, dry guitar signal; it's purely the reverb" (DiPerna 1992). 
The application of time-based signal processors to the guitars and vocals is evident from the moment the track begins. The reverse reverb to which Shields refers appears to have been applied to all the guitars throughout "I Only Said" even the overdubbed, lead riff (00:00-00:21). This is ascertained by the absence of any immediate attack in the sound envelopes, which is precisely the "growing out of nowhere" sound that Sangild perceived. To that end, the prominent exposure of effected sound in relation to the original results in the lead guitar riff sounding more like notes bowed as opposed to plucked. The reverse reverb effects are prominent at 01:48 and 01:51, and again between 02:09-02:15, where the reversed envelopes of guitar incidentals are clearly audible.

The strummed guitars (of which there are at least two) feature plenty of distortion and tremolo, along with a warping effect which is a signature motif of Shields' playing style, achieved by simultaneously bending the tremolo arm on the guitar as he strums (DiPerna 1992; McGonigal 2007). However, whilst these tremolo arm effects impact on pitch, lending the entire track a discordant aesthetic, the sense of impenetrable depth is in no small part due to the presence of time-based signal processors. Each strummed chord appears to morph into the next, which is further exacerbated by the tremolo. Whilst each strum is audible, defined attacks are missing, which is indicative of reverse reverbs. This is most evident - and arguably, has the biggest impact on the track's overall shape - as the track moves from verse two into an extended coda at 03:18. At this point, the addition of further guitar tracks as well as an increase in overall volume has boosted the track's overall shape with a notable increase in textural density.

Of the many musical and timbral components to "I Only Said", the vocals play a key role in the track's overall shape, particularly as it changes over time. The vocals are submerged beneath the layers of guitar tracks, which completely obscure the lyrical narrative. This deliberate foregrounding of impenetrable, discordant instruments over the vocals is another of My Bloody Valentine's signature motifs. The apparent blending of words into the musical accompaniment suggests they have been processed in similar ways to the guitars; in terms of timbre, the vocal features similar, although lighter, distortion. Whilst sibilant sounds are prominent in the vocal delivery, the words lack the defining attack component of their natural envelope, suggesting they have been heavily processed with reverb, with more of the reverberant signal than original audible in the mix. This further renders the lyrical components indecipherable and forces the listener into confronting the track's condensed, foregrounded discordance and distortion. These aspects are considered further in the discussion below.

\section{Discussion}

So far, I have focused on the "what" in terms of research context, time-based signal processors, and case study material. As Allan Moore suggested (2012), more important is the "so what?" - and so here are some findings.

Whilst all four case study examples feature distinctive applications of reverb, it is important to acknowledge the historical trajectory of time-based processing in popular music. With regard to "Just Like Honey" and "I Only Said", both strongly 
allude to Phil Spector's 'Wall of Sound' technique - the burying of instruments in a wash of ambience that was such an iconic feature of his work in the 1960s. This is a notable and instantly recognisable production technique that, to an extent, was applied in an exaggerated form to the indie, alternative, and shoegaze music of the post-punk era as heard in these two tracks. That being said, there are fundamental differences in both the application and consequences of time-based processing. For Spector, it was a clear fabrication of space - the situating of instrumental mass in a large, hall-like situ. Despite the presence of the so-called "wall", instruments were clearly sonically discernible. In the case of "I Only Said", it is not possible to ascertain the number of instruments and voices present in the mix, due to the concealment of the vocal recordings and dense, multilayered guitars. For the Jesus and Mary Chain, the reverb forms only part of their overall sonic canvas; it is the consolidation of plate reverb with fuzzy distortion that is integral to their musical identity, as opposed to the reverb on its own. To that end, for both these tracks, the application of time-based signal processing goes far beyond straightforward space fabrication and becomes embodied in their very musical identity. To put it another way, both the Jesus and Mary Chain and My Bloody Valentine would sound entirely different without time-based processors. Of course, this could be true of any number of artists, as reverb in particular is so ubiquitous in contemporary recorded popular music. However, in both examples, time-based signal processing means more than a fabrication of space, embellishment, or indeed, simply a standard recording process; it is an instrument in its own right, a tool for musical - particularly textural - construction and shaping. "Blue Flower" is another nod to sound processing in the 1960s, albeit in a different way. The reverb used by the Velvet Underground was heady, smoky, and opaque in nature, designed in part to emulate alternate states of mind and consciousness. Stephen Mallinder describes tangential, "alternative" sounds present in avant-rock music at the turn of the 1970s, including those present in the music of the Velvet Underground. He states: "Importantly, there was no real desire to separate the ambience or cacophony from the music structures which carried the songs themselves on the part of these music makers" (2013: 86).

In "Blue Flower", we hear not simply a decision to separate ambience from musical structures, but the other way around. We hear the bold, deliberate construction of a much larger space than the small, female voice located within it: the ambient space constructed from time-based signal processors is consciously foregrounded in the track and, therefore, becomes one of the most important aspects of it. We can perhaps deduce from Mallinder's point that where reverberant spaces existed in popular song of the 1960s, by the 1980s these spaces were actively and intentionally constructed. However, whilst Mallinder refers to the inextricability of musical elements and overall ambience on a macro level, time-based signal processors can be effectively used to create micro spaces within individual tracks.

Whilst "The Killing Jar" features significant time-based processing, the chorus effect in particular has been used in a creative way to construct the shape of two alternate sound spaces. Here, Moore's use of Johnson's containment schema (Moore 2012) is useful: the lyrical narrative features a body of water, the "twisted roots" of a tree or plant, and a "wishing well", suggesting we are in an outside environment. Indeed, the use of lengthy reverbs across multiple instruments 
substantiates this as an outdoor setting. The central theme, however, is that of "The Killing Jar". This container, in which an insect is suffocated, is clearly constructed with the foregrounded, chorus-processed bass guitar in verse two, thus shaping two sonically distinctive spaces within the same track. This brings me to another point of discussion: the interplay between the time-based signal processors and other elements of the recordings, including perceived depth. Interestingly, the effect on depth of field is entirely different in all four case studies, which is a result of the interplay between the respective reverberation and other factors such as dynamic range, spatial position, and the relative position of each instrument in the mix. For example, the presence of repetitive reverse gated reverbs in "I Only Said" creates a claustrophobic, contained space for the instrumentation. Coupled with the directly centred, mono position, as a track "I Only Said" is narrowly defined spatially. The position of the reverberant vocal much lower in the mix certainly submerges the voice(s) underneath the noisy instrumental wash. However, it is the reverse reverb that shapes the recording more than anything else; this deliberate temporal manipulation disorients the instrumentation - particularly against the drums and bass, which feature little to none of the extensive processing of the guitars and vocals - resulting in a circular, drowning effect as two sets of instruments pull the track into two seemingly opposite directions. Its relentless nature for the entirety of the track (lasting more than 05:30) creates what Blake perceptively describes as a "literally indigestible wall of sound violently projected a discomfiting motility" (Blake 2012). Conversely, the application of time-based processing in "The Killing Jar" has the very opposite effect. In this track, the instruments are separated around the stereo field: the cello is positioned centre right, güiro hard right, triangle (or triangle-like instrument) hard left, with bass, drums, and vocals centred and cymbal overheads panned left and right. Despite the audible reverb across all drums, vocals, and percussion instruments, and a chorus effect on bass and guitar, the track is not "muddied" or particularly texturally dense. Instead, the processing has a greater effect on the breadth of the track, situating the instruments in a wide, panoramic soundscape. From "I Only Said" and "The Killing Jar", we can therefore deduce that multiple instrumental applications of time-based signal processing will have a greater effect on textural depth and density - and, therefore, the track's overall shape - if the recording is mono than if it is stereo, as Kevin Shields points out:

Everything I did is mostly mono ... there's no set area of separation. The sense of bigness just comes from the depth of perception. The classic 1980s version of stereo was basically a drum sound that's really widened by stereo effects and gated, and the guitars are really panned to extremes and it's just vocals and drums in the middle with overdubs. It was a corporate, weak sound. (McGonigal 2007: 49-50)

Here, Shields acknowledges the perceptual, multi-dimensional sound space that Dockwray and Moore elucidate in their work. By suggesting that a maximized stereo field and ambience are associated with a "corporate, weak sound", he is associating the clean "clear" sounds of spatially diverse 1980s recordings with the commercial mainstream. Therefore, by situating his instruments in a mono sound field with unique applications of time-based processors, he has created something 
altogether alternative, befitting My Bloody Valentine's independent label association and the wider business and philosophical aesthetics of independent music.

In both "Just Like Honey" and "Blue Flower", the use of reverb is equally heavy and prominent - and certainly more recognisable as reverb - but the recordings are shaped entirely differently. In the case of "Just Like Honey", the coupling of the reverb, featuring an extraordinarily long decay time, with top-end heavy, fuzzy cymbals and distortion-laden guitars has situated the track in a tinny, metallic space like a warehouse or aircraft hangar. Regardless of the application of a then-vintage EMT Plate reverberator and the more recent AMS RSX-16, the result is a space that dwarfs the instruments and voice situated within it. The Jesus and Mary Chain are, therefore, in an entirely unrealistic space, bearing little resemblance to standard rooms, halls, or cathedrals that mechanical or digital reverberators are designed to emulate. The same can be said for "Blue Flower", but the significant difference is the treatment of the vocals compared to the rest of the instrumentation; they are separated, with Hope Sandoval's vocal featuring an entirely different reverb than the instruments. Regardless of the fact that her voice is situated in the same track as the guitars, drums and bass, her vocal is isolated and this has two major consequences: firstly, it situates her voice as the most important instrumental focal point of the track; and secondly, it implies loneliness, detachment, and a sense of distance from both the other musicians in the band and the main protagonist featured in the lyrical narrative of "Blue Flower". Bannister (2006a, 2006b) recognized the potential of large, reverberant spaces to indicate such feelings, although in the case of both "Just Like Honey" and "Blue Flower", the vast reverbs not only indicate and reinforce such emotions, but exacerbate them.

In all these examples, we hear instruments transformed and shaped from their original sound. The final, composite sound image is shaped by a blend of the original instrument signal and the applied time-based signal processing into an altogether constructed sound image. The case studies featured in this article certainly fall at the extremities of time-based signal processing on recordings of the era.

\section{Summary}

In summary, time-based signal processors are used in more complex ways than to simply fabricate space. They can simulate both real and imaginary spaces, create alternate sound worlds, and impact greatly on textural and spatial attributes of a recording, thus greatly influencing a track's overall shape. Aside from "The Killing Jar", none of these tracks were aimed at the commercial mainstream, and as such, the featured production attributes can be considered equally as alternative as the musical ones. We can, therefore, consider these examples as rejections of commercial music production values. The sonic canvases are opposed to the intentional - and aspirational - clarity associated with then-modern, commercial rock record production. That being said, there is a clear sonic continuum present in all these examples that can be traced back to a so-called "golden age" of record production where creative recording and processing techniques, such as 
those used by Phil Spector, Joe Meek and Brian Wilson, featured on numerous successful commercial popular music recordings.

Acoustically realistic representations of space are neither the intention nor the outcome here - in all examples, the instruments and voice are situated in alternative spaces to the rooms, halls, and cathedrals so often emulated in timebased processing. Throughout these case studies, the usually micro application of time-based processing is foregrounded in the recording as a macro feature. Take the processing away, and the tracks would not retain their temporal attributes; they would be significantly texturally diminished and, therefore, would not convey the same overall meaning. In these examples we hear time-based signal processing as no less integral to artists' musical identities than any other musical element, instrument, or performance. The foregrounding of reverbs, echoes, delays and chorus effects in the overall musical canvas presents a challenge to production values commensurate with the commercial mainstream and is, therefore, intrinsic to alternative musics.

\section{Endnotes}

${ }^{1}$ The Association for the Study of the Art of Record Production hosts annual conferences and oversees a journal: The Journal on the Art of Record Production. Further information can be found at: www.arpjournal.com.

${ }^{2} \mathrm{An}$ "echo" is a natural phenomenon whereby a reflection of sound is heard after a direct sound. Fabricated echo effects can be short single "slap-back" style reflections or longer, repetitive reflections designed to emulate a space such as a subway tunnel.

3 "Reverb" is used in this article as an abbreviation for "reverberation". Reverberation is a natural phenomenon heard as the build-up of multiple sound reflections as they decay in amplitude following a direct sound. In time-based signal processing, common fabricated reverb parameters include: emulations, either of physical spaces (room, hall, and cathedral are common settings) or mechanical systems (including plates and springs); pre delay time (often in $\mathrm{ms}$ and used to separate the direct sound from its first, early reflections); decay time (the time it takes for the reverberant tail to decay to $0 \mathrm{~dB}$ ); and, diffusion (density of reflections in early reflections and reverberant tail).

4 "Delay" is a time-based signal processor that holds an input signal, feeding it back into the original after a set time period. Common delay parameters include: delay time (the length of time between the onset of the original signal and its delayed signal); and, feedback (the number of delays or repeats, often depicted as a feedback percentage).

${ }^{5}$ A "chorus" effect is similar to a delay. The effect is achieved by mixing a delayed, pitchmodulated copy of a signal with its original. The effect creates a thicker, often "wider" sound, where multiple sounds are perceived as originating from a single sound source.

\section{References}

Bibliography

Alleyene, M. 2008. Globalization and Commercialization of Caribbean Music.

Popular Music History 3(3): 247-273. 
Bannister, M. -

2006a. "Loaded": Indie Guitar Rock, Canonism, White Masculinities. Popular Music 25(1): 77-95.

2006b. White Boys, White Noise: Masculinities and 1980s Indie Guitar Rock. Farnham: Ashgate.

Bartlett, B. and Bartlett, J. 2012. Practical Recording Techniques. Oxon: Focal Press.

Bennett, S. -

2009. Revolution Sacrilege! Examining the Technological Divide Among Record Producers in the Late 1980s. Journal on the Art of Record Production 4. www.arpjournal.com; Accessed: 29 December 2017.

2015. Never Mind the Bollocks... A Tech-Processual Analysis. Popular Music and Society 38(5): 466-486.

2016. Songs About Fucking: Southern Studios and the Construction of Punk's Subversive Sonic Signature. Keep It Simple, Make It Fast - DIY Cultures, Spaces, Places. International conference. Porto: Universidade do Porto.

Bennett, S. and Moore, A.F. 2012. Tech-Processual Shaping of Recorded Popular Song. Music and Shape Conference. AHRC Research Centre for Musical Performance as Creative Practice. London: King's College.

Blake, D.K. 2012. Timbre as Differentiation in Indie Music. MTO - A Journal of the Society for Music Theory 18 (2)

http://www.mtosmt.org/issues/mto.12.18.2/mto.12.18.2.blake.html; Accessed: 1 March 2016.

Burgess, R.J. 2005. The Art of Music Production. London: Omnibus Press.

Cottrell, S. 2010. The Rise and Rise of Phonomusicology. In A. Bayley Ed. Recorded Music: Performance, Culture and Technology. Cambridge: Cambridge University Press: 15-36.

Crane, L. 2009. Tape Op: The Book About Creative Music Recording Vol. II. Milwaukee, WI: Hal Leonard.

Cunningham, M. 1998. Good Vibrations. London: Sanctuary Music Publishing.

Dibben, N. 2009. Vocal Performance and the Projection of Emotional Authenticity. In D.B. Scott Ed. The Ashgate Research Companion to Popular Musicology. Farnham: Ashgate: 317-334.

DiPerna, A. 1992. Bloody Guy. Guitar World. March 1992. http://www.tohereknowswhen.org/press/guitarworld-mar92.html; Accessed: 1 March 2016.

Dockwray, R. and Moore, A. F. 2010. Configuring the Sound-Box: 1965-1972. Popular Music (29)2: 181-197.

Doyle, P. 2005. Echo and Reverb: Fabricating Space in Popular Music Recording 1900-1960. Middletown, CT: Wesleyan University Press.

Everett, W. Ed. 2000. Expression in Pop-Rock Music. A Collection of Critical and Analytical Essays. New York: Garland Publishing.

Hall, E. 1966. The Hidden Dimension. New York: Doubleday.

Hodgson, J. 2010. Understanding Records: A Field Guide to Recording Practice. New York: Bloomsbury Academic.

Howard, D. 2016. The Creative Echo Chamber: Contemporary Music Production in Kingston, Jamaica. Kingston: Ian Randle Publishers. 
Izhaki, R. 2008. Mixing Audio: Concepts, Practices and Tools. Boston: Focal Press.

Johnson, M. 1987. The Body in the Mind. Chicago, IL: Chicago University Press. Kahlin, D. and Ternström, S. 1999. The Chorus Effect Revisited. Experiments in Frequency-Domain Analysis and Simulation of Ensemble Sounds. Milan: Proceedings of the $25^{\text {th }}$ EUROMICRO Conference: 75-80.

Katz, M. 2004. Capturing Sound: How Technology Has Changed Music. Berkeley, CA: University of California Press.

Kehew, B. and Ryan, K. 2006. Recording The Beatles. USA: Curvebender Publishing.

Lacasse, S. 2003. Reverb. In J. Shepherd et al. Eds. 2003. Continuum Encyclopedia of Popular Music of the World: Volume II-Performance and Production. London and New York: Continuum: 227-228.

Machin, D. 2010. Analysing Popular Music: Image, Sound and Text. London: Sage.

Mallinder, S. 2013. Sounds Incorporated: Dissonant Sorties into Popular Culture. In: M. Goddard, B. Halligan, and N. Spelman Eds. Resonances: Noise and Contemporary Music. New York: Bloomsbury Academic: 81-94.

McDonald, C. 2000. Exploring Modal Subversions in Alternative Music. Popular Music 19(3): 355-63.

McGonigal, M. 2007. My Bloody Valentine's Loveless. New York: Bloomsbury Academic.

Milner, Greg. 2009. Perfecting Sound Forever: The Story of Recorded Music. London: Granta.

Moore, A. 1993. Analyzing Popular Music. Farnham: Ashgate.

2001. Rock: The Primary Text. Aldershot: Ashgate. Second edition.

2012. Song Means: Analyzing and Interpreting Recorded Popular Song.

Farnham: Ashgate.

Sangild, T. 2011. The Aesthetics of Noise by Torben Sangild. Extravagentment. https://extravagantment.wordpress.com/2011/12/08/the-aesthetics-of-noise-bytorben-sangild/: Accessed: 1 March 2016.

Tagg, P. 2000. "The Work": An Evaluative Charge. In M. Talbot Ed. The Musical Work. Reality or Invention? Liverpool: Liverpool University Press: 153-167.

Uncut. 1999. Uncut magazine Interviews Tony Visconti. Uncut. http://www.bowiegoldenyears.com/low.html; Accessed: 1 March 2016.

Zak, A.J. III 2001. The Poetics of Rock: Cutting Tracks, Making Records. Berkeley, CA: University of California Press.

\section{Discography}

Bowie, David. 1977. Low. RCA Victor, UK.

Jesus and Mary Chain, the. 1985. "Just Like Honey". Psychocandy. Blanco Y

Negro, UK.

Mazzy Star. 1990. "Blue Flower". She Hangs Brightly. Capitol Records, US.

Mazzy Star. 1993. So Tonight That I Might See. Capitol Records, US. 
Mazzy Star. 1996. Among My Swan. Capitol Records, US.

My Bloody Valentine. 1991. "I Only Said". Loveless. Creation Records, UK. Ronettes, the. 1963. "Be My Baby". Philles Records, US.

Sex Pistols. 1977. Never Mind The Bollocks... Here's The Sex Pistols. Virgin, UK. Siouxsie and the Banshees. 1988. "The Killing Jar". Peepshow, Polydor, UK. Slapp Happy. 1972. "Blue Flower". Sort Of. Polydor, UK.

Velvet Underground, the. 1967. The Velvet Underground and Nico. Verve Records, US. 